\title{
Improvement of the Direct Modulation Behavior of Semiconductor Lasers by Using a Holding Beam
}

\author{
G. Morthier, Senior Member, IEEE, and B. Moeyersoon, Student Member, IEEE
}

\begin{abstract}
We show theoretically that the direct modulation properties of semiconductor lasers can be significantly improved by the injection of a so-called holding beam-a continuous-wave beam at the transparency wavelength of the gain medium. Both the small-signal and the large-signal behavior are investigated.
\end{abstract}

Index Terms-Laser modulation, optical pulse shaping, semiconductor lasers, simulation.

\section{INTRODUCTION}

$\mathbf{S}$ EMICONDUCTOR lasers under direct modulation usually suffer from strong relaxation oscillations, which can cause relatively long and undesired transients during ON-OFF switching and are accompanied by an equally undesired frequency chirping. For long-haul optical communications, these problems have been overcome by making use of external modulators. However, direct modulation is still considered to be a preferred solution for access and metro optical networks, where low cost is of paramount importance [1].

Recently, it has been proposed to remove the undesired transients by pulse shaping of the injected current [2]. This method is very effective but it obviously requires very advanced electronic drivers able to do pulse shaping at very high bitrates of $10 \mathrm{~Gb} / \mathrm{s}$ or more. Moreover, the specific shaping of the current pulses depends on the current or power levels used for the digital zeroes and ones. The method we propose here, using a so-called holding beam, only requires the use of an external continuous-wave $(\mathrm{CW})$ light beam. And it is independent of the other modulation details. Our method resembles earlier work (e.g., [3], [4]), where the injection of a beam with wavelength close to the lasing wavelength or injection locking are used as methods to improve the laser dynamics. Both methods also affect the other laser parameters such as the output power and wavelength and/or are very difficult to achieve in a stable manner. The method presented here specifically relies on the injection of a beam at the transparency wavelength and, therefore, does not affect the other laser characteristics.

The use of a holding beam was proposed several years ago to improve the dynamic behavior of semiconductor optical amplifiers [5], [6]. The technique, consisting of the injection of a $\mathrm{CW}$ light beam at the transparency wavelength of the gain medium, allows us to reduce the differential carrier lifetime without affecting the total stimulated emission rate or carrier depletion.

Manuscript received September 15, 2003; revised March 26, 2004.

The authors are with the Department of Information Technology, Ghent University - IMEC, B-9000 Gent, Belgium (e-mail: morthier@intec.UGent.be).

Digital Object Identifier 10.1109/LPT.2004.829534
In this letter, we show that the same technique can be used to improve the dynamic behavior of semiconductor lasers. As will be shown, the injection of the holding beam does not increase the modulation bandwidth or resonance frequency itself, but results in a significant increase of the damping of the relaxation oscillations. Under direct, large signal modulation, it leads to significantly reduced transient effects and reduced chirping, even at low bias.

\section{THEORY}

The method can easily be explained using the standard rate equations for the average carrier density $N$ in the active layer and the photon number $S_{l}$ of the laser field

$$
\begin{aligned}
& \frac{d S_{l}}{d t}=\left[\Gamma\left(A_{l} N-B_{l}\right)\left(1-\varepsilon S_{l}\right)-\frac{1}{\tau_{p}}\right] S_{l}+R_{\text {spon }} \\
& \frac{d N}{d t}=\frac{I}{q V}-\frac{N}{\tau_{N}}-\Gamma\left(A_{l} N-B_{l}\right)\left(1-\varepsilon S_{l}\right) \frac{S_{l}}{V}-\Gamma\left(A_{t} N-B_{t}\right) \frac{S_{\mathrm{inj}}}{V}
\end{aligned}
$$

with $I$ the injected current, $V$ the volume of the active layer, $\Gamma$ the confinement factor, $\tau_{P}$ the photon lifetime, and $\tau_{N}$ the carrier lifetime. $A_{l}, B_{l}, A_{t}$, and $B_{t}$ are the gain parameters at the lasing wavelength and at the transparency wavelength, respectively, and obviously $A_{t} N-B_{t}=0$ in steady state. $R_{\text {spon }}$ is the spontaneous emission coupling into the laser mode and $S_{\text {inj }}$ is the number of photons in the laser cavity due to optical injection. Gain suppression $(\varepsilon)$ for the gain at the transparency wavelength has been neglected for simplicity.

Due to $A_{t} N-B_{t}=0$, the steady state solution of the rate equations is unchanged. However, the dynamic behavior is modified and the differential carrier lifetime becomes dependent on the injected power. From the small signal solution of the rate equations under modulation at frequency $f$, one finds that the intensity modulation is a second-order response, characterized by a resonance frequency (the resonance frequency of the relaxation oscillations) and a damping. The damping of the relaxation oscillations is given by

$$
\vartheta=\frac{1}{\tau_{N}}+\Gamma A_{l} \frac{S_{l}}{V}+\Gamma A_{t} \frac{S_{\mathrm{inj}}}{V}+\frac{R_{\mathrm{spon}}}{S_{l}}+\varepsilon \Gamma\left(A_{l} N-B_{l}\right)\left(1-\varepsilon S_{l}\right) S_{l} .
$$

For large $S_{\text {inj, }}$, this damping can be significantly larger than without holding beam. Moreover, it is independent of the bias of the laser diode; i.e., even at low bias current, where the relaxation oscillations are normally little damped, the damping remains significant. The resonance frequency itself is hardly affected by the holding beam. 


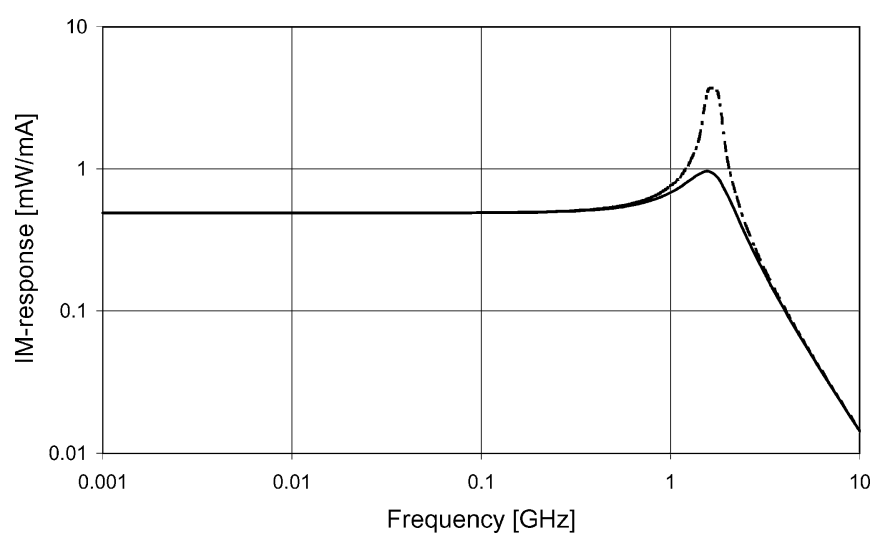

Fig. 1. Small signal intensity modulation response for a $\lambda / 4$-shifted DFB laser at $1-\mathrm{mW}$ output power with a holding beam of $20 \mathrm{~mW}$ (full line) and without holding beam (dashed line). More laser parameters are given in the text.

It can be remarked that the increase of the damping due to a holding beam will limit the maximum available 3-dB direct modulation bandwidth of a laser diode. However, in practice, this theoretical maximum can only be achieved at very high bias levels and lasers are typically used at lower bias levels. As we will show with a few numerical examples, the modulation response at not too high bias levels can significantly be improved when a holding beam is applied. The ON-OFF switching no longer exhibits strong relaxation oscillations while a similar maximum speed can be maintained.

It can be remarked that the relation between chirp $\Delta \nu$ and power $P(t)$ remains unchanged, i.e., from the linearization of the rate equation for $S_{l}$ one readily finds

$$
\Delta \nu=\frac{\alpha}{4 \pi} \frac{d(\ln P)}{d t}+\kappa P(t)
$$

with $\alpha$ the linewidth enhancement factor. Since the (transient) power variations $P(t)$ are much smaller with the use of a holding beam, the chirp (both the adiabatic and the dynamic chirp) will also be much smaller.

\section{NUMERICAL RESULTS}

Fig. 1 shows the influence of a holding beam on the small signal response of a $400-\mu \mathrm{m}$-long $\lambda / 4$-shifted distributed feedback (DFB) laser with $\kappa L=1$. A holding beam of $20 \mathrm{~mW}$ was injected at the transparency wavelength of $1.483 \mu \mathrm{m}$. The lasing wavelength was $1.5545 \mu \mathrm{m}$ and the wavelength-dependent gain was modeled as outlined in [7]. Fig. 1 shows the small signal intensity modulation response at a bias output power of $1 \mathrm{~mW}$. One can see that at this low bias level the relaxation oscillations are significantly damped due to the holding beam. However, the $3-\mathrm{dB}$ bandwidth is almost unaffected.

To see the influence on the large signal modulation, we have done time-domain simulations using our model CLADISS [7]. The 400- $\mu \mathrm{m}$-long $\lambda / 4$-shifted DFB laser (with threshold current $13.65 \mathrm{~mA}$ ) used in the small signal calcuation was modulated digitally at $1 \mathrm{~Gb} / \mathrm{s}$ between 14 and $30 \mathrm{~mA}$. Fig. 2 shows the power versus time in the lasing mode for a holding beam of 10 , 20 , and $50 \mathrm{~mW}$. One can see that the relaxation oscillations are damped more as the holding beam power increases. At the same time, however, the modulation bandwidth decreases somewhat

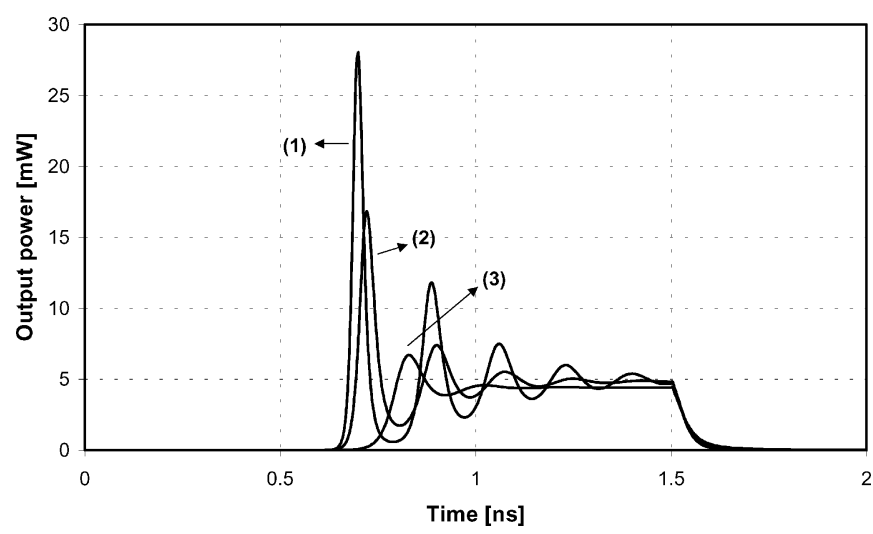

Fig. 2. Power versus time for a 1-Gb/s ON-OFF modulation of the laser of Fig. 1 for a holding beam of $10(1), 20(2)$, and $50 \mathrm{~mW}$ (3). The rising edge of the current pulse starts at time $0.5 \mathrm{~ns}$.

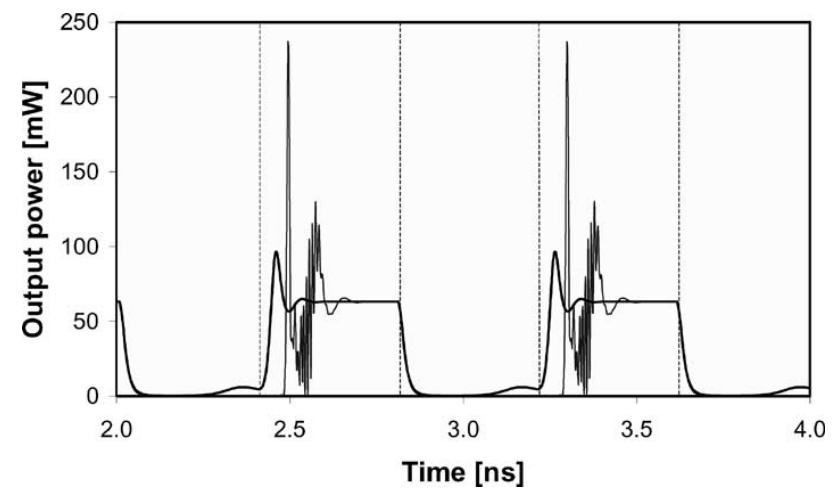

Fig. 3. Power versus time for a $2.5-\mathrm{Gb} / \mathrm{s}$ ON-OFF modulation of a very fast two-section DBR laser: without holding beam (thin line) and with a holding beam of $20 \mathrm{~mW}$ (thick line). The vertical dashed lines indicate the switching times.

and the switch-ON time increases. This last effect is also due to the damping of the relaxation oscillations. The switch-ON furthermore seems to start later as the damping increases. In reality, the switch-ON starts at the same moment (at $t=0.5 \mathrm{ns)}$ for all three curves in Fig. 2 and the delay is due to an exponential rise of the power from very low values to values that are visible in the figure. However, since this rise is part of the first period of the relaxation oscillations, it is again slower for increasing holding beam power. One can finally notice that the holding beam does not seem to influence the switching-OFF of the laser. The reason for this is that the first stage of the OFF-switching occurs in a time interval much shorter than the carrier lifetime and its duration is, thus, unaffected by a modification of the carrier lifetime using a holding beam.

We also considered a very fast two-section DBR laser (with a $120-\mu \mathrm{m}$ cleaved active section and a $450-\mu \mathrm{m}$-long Bragg section with $\kappa L=1.35)$. Fig. 3 shows the power versus time in the lasing mode without holding beam and with a $20-\mathrm{mW}$ holding beam. The laser has a threshold current of $9.5 \mathrm{~mA}$ and was digitally modulated at $2.5 \mathrm{~Gb} / \mathrm{s}$ between 10 and $150 \mathrm{~mA}$. In [8], it has been shown that this two-section DBR laser has two modulation resonance frequencies and this is clearly visible in the time dependence of the power without holding beam. It can be seen in Fig. 3 that the relaxation oscillations are much less pronounced with the holding beam. However, the holding 
beam also prevents the laser gain from decreasing far below the threshold gain during the switching OFF. The power during the OFF-period is, therefore, two to three orders of magnitude larger with the holding beam. For this reason, no delay accompanies the switching-ON as is the case without holding beam. As mentioned above, the smaller power variations also imply a much smaller chirp.

It can be noticed that rather high power levels are required for the holding beam. Typically, $20 \mathrm{~mW}$ has to be injected into the laser diode, which means that the laser diode delivering the holding beam will have to produce $50 \mathrm{~mW}$ or more. However, such power levels can be obtained with current laser diodes at many wavelengths; e.g., for 1550-nm laser diodes, the holding beam is typically in the 1480-1510-nm range and pump lasers in this wavelength range with high power levels have been fabricated for Raman or Erbium-doped fiber amplifiers.

Finally, we also remark that the low power levels for the logical " 0 " in both Figs. 2 and 3 are due to the switching-OFF transient, i.e., the carrier density is temporarily below the threshold value and the power level still decreases. These power levels are, therefore, below the steady state levels corresponding with the OFF-currents (which are above threshold).

\section{CONCLUSION}

We have shown, by using small-signal analysis as well as large signal time domain simulations, that the modulation response of laser diodes can be improved using the injection of
CW light at the transparancy wavelength. The relaxation oscillations are damped much more when a holding beam is applied, while the modulation bandwidth remains virtually unaffected. Under large signal modulation, this results in much smaller power fluctuations and, as a direct consequence, also much smaller chirp.

\section{REFERENCES}

[1] K. Sato, "Semiconductor light sources for 40-Gb/s transmission systems," J. Lightwave Technol., vol. 20, pp. 2035-2043, Dec. 2002.

[2] N. Dokhane and G. L. Lippi, "Improved direct modulation technique for faster switching of diode lasers," in Proc. Inst. Elect. Eng., Optoelectronics, vol. 149, 2002, pp. 7-16.

[3] R. Lang and K. Kobayashi, "Suppression of the relaxation oscillation in the modulated output of semiconductor lasers," IEEE J. Quantum. Electron., vol. 12, pp. 194-199, Mar. 1976.

[4] J. Dellunde, M. C. Torrent, J. M. Sancho, and M. SanMiguel, "Frequency dynamics of gain-switched injection-locked semiconductor lasers," IEEE J. Quantum Electron., vol. 33, pp. 1537-1542, Sept. 1997.

[5] M. A. Dupertuis, J. L. Pleumeekers, T. P. Hessler, P. E. Selbmann, B. Deveaud, B. Dagens, and Y. Y. Emery, "Extremely fast high-gain and low-current SOA by optical speed-up at transparency," IEEE Photon. Technol. Lett., vol. 12, pp. 1453-1455, Nov. 2000.

[6] T. P. Hessler, M. A. Dupertuis, B. Deveaud, J. Y. Emery, and B. Dagens, "Optical speedup at transparency of the gain recovery in semiconductor optical amplifiers," Appl. Phys. Lett., vol. 81, pp. 3119-3121, 2002.

[7] P. Vankwikelberge, G. Morthier, and R. Baets, "CLADISS- A longitudinal multimode model for the analysis of the static, dynamic and stochastic behavior of diode lasers with distributed feedback," IEEE J. Quantum Electron., vol. 26, pp. 1728-1741, Oct. 1990.

[8] G. Morthier, R. Schatz, and O. Kjebon, "Extended modulation bandwidth of DBR and external cavity lasers by utilizing a cavity resonance for equalization," IEEE J. Quantum Electron., vol. 36, pp. 1468-1475, Dec. 2000. 